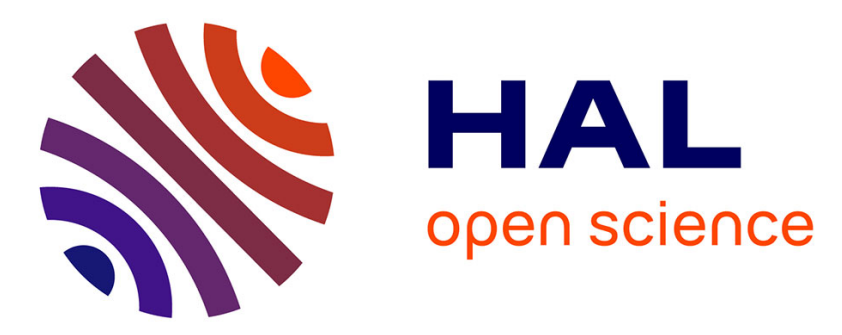

\title{
Faire face à des questions de travail : les leviers mobilisés par des éleveurs laitiers
}

Nathalie Hostiou, Sophie Chauvat, Sylvie Cournut

\section{To cite this version:}

Nathalie Hostiou, Sophie Chauvat, Sylvie Cournut. Faire face à des questions de travail: les leviers mobilisés par des éleveurs laitiers. L'agriculture en famille: travailler, réinventer, transmettre, 2014, 10.1051/978-2-7598-1192-2.c008 . hal-02087409

\section{HAL Id: hal-02087409 \\ https://hal.uca.fr/hal-02087409}

Submitted on 2 Apr 2019

HAL is a multi-disciplinary open access archive for the deposit and dissemination of scientific research documents, whether they are published or not. The documents may come from teaching and research institutions in France or abroad, or from public or private research centers.
L'archive ouverte pluridisciplinaire HAL, est destinée au dépôt et à la diffusion de documents scientifiques de niveau recherche, publiés ou non, émanant des établissements d'enseignement et de recherche français ou étrangers, des laboratoires publics ou privés. 


\title{
Faire face à des questions de travail : les leviers mobilisés par des éleveurs laitiers
}

\author{
Nathalie Hostiou ${ }^{1}$, Sophie Chauvat ${ }^{2}$, Sylvie Cournut ${ }^{3}$
}

\section{Introduction}

Les questions de travail en élevage laitier deviennent essentielles pour traiter du devenir de ce secteur. Le travail est devenu un frein à l'installation et au maintien des éleveurs dans cette activité (Ferris et al., 2006 ; Madelrieux et Dedieu, 2008). Depuis les années 1970, en Europe, le nombre d'exploitations laitières et la maind'œuvre dans les élevages ne cessent de diminuer fortement (Gambino et al., 2012), alors que les structures s'agrandissent (Seegers et al., 2006). Par exemple, le nombre de vaches laitières a augmenté de $36 \%$ entre 2000 et 2010. En France, les collectifs de travail gérant des exploitations laitières se diversifient. Le modèle d'exploitation géré par deux unités de travail annuel (UTA), c'est-à-dire le chef d'exploitation et son conjoint et/ou un aide familial, laisse la place à une réalité plus diversifiée : des exploitants seuls permanents, des couples, des exploitations familiales avec du salariat permanent, des formes associatives gérant des regroupements d'exploitations ou du salariat partagé (Cochet, 2008 ; Hervieu et Purseigle, 2013). Ces évolutions des collectifs de travail font partie des scénarios d'avenir de l'activité d'élevage. Ces mutations structurelles s'accompagnent aussi de changements importants dans les rapports des éleveurs à leur travail. Les attentes d'un travail maîtrisé, distinct de la vie familiale, et la volonté de se préserver du temps libre prennent le pas sur le «labeur paysan" (Barthez, 1986), où vie privée et travail sont confondus.

1. INRA, UMR 1273 Métafort, 63122 Saint-Genès Champanelle, France ;

AgroParisTech, UMR Métafort, 63170 Aubière, France ;

Clermont Université, VetAgro Sup, UMR Métafort, 63370 Lempdes, France ;

Irstea, UMR Métafort, 63170 Aubière, France ; nhostiou@clermont.inra.fr

2. Institut de l'élevage, Supagro, 34060 Montpellier, France, Sophie.Chauvat@idele.fr

3. Clermont Université, VetAgro Sup, UMR Métafort, 63370 Lempdes, France ;

INRA, UMR 1273 Métafort, 63122 Saint-Genès Champanelle, France ;

AgroParisTech, UMR Métafort, F-63170 Aubière ; Irstea, UMR Métafort, 63170 Aubière,

France, sylvie.cournut@vetagro-sup.fr

Ce chapitre de l'ouvrage L'agriculture en famille : travailler, réinventer, transmettre est publié en Open Access sous licence creative commons CC-BY-NC-ND permettant l'utilisation non commerciale, la distribution, la reproduction 
Ces évolutions s'observent dans le Ségala, région rurale naturelle située à cheval sur les départements de l'Aveyron, du Cantal et du Lot, qui se caractérise par une densité agricole très forte et une orientation affirmée vers la production laitière. Les exploitations laitières de taille moyenne (220 000 litres de quota en moyenne) restent familiales malgré une diversification des formes de collectifs. Les organisations professionnelles de cette région s'intéressent de plus en plus à la problématique du travail, faisant écho à la fois aux questions posées par les éleveurs par rapport à leur travail mais aussi aux inquiétudes des acteurs de la filière quant au devenir de cette activité. Les organismes de conseil sont demandeurs de connaissances et d'outils pour accompagner les réflexions des éleveurs sur l'adaptation de leur exploitation du point de vue de l'organisation du travail.

Pour réorganiser le travail sur leur exploitation, les éleveurs mettent en place des solutions relevant de trois grands leviers (Dedieu et Servière, 2001). Ils peuvent simplifier la conduite du troupeau et des surfaces fourragères par exemple avec la monotraite (Rémond et Pomiès, 2007) ou la diminution du nombre de distribution de la ration (Ferris et al., 2006). La main-d'œuvre intervenant sur l'exploitation peut être recomposée, par exemple par l'embauche de salariés ou l'externalisation auprès d'entreprises agricoles (Hadley et al., 2002). Les éleveurs peuvent également améliorer les bâtiments et équipements en investissant, par exemple dans du matériel de traite (Wagner et al., 2001 ; Rotz et al., 2003) ou pour l'alimentation (Kung et al., 1997). Des études ont montré que ces solutions ne répondaient pas aux mêmes attentes (Moreau et al., 2004) et n'étaient pas indépendantes les unes des autres (Parsons et al., 2004 ; Cournut et Dedieu, 2005). Par exemple, la monotraite est souvent associée aux vêlages groupés, à l'arrêt de la traite sur une période et au recours à l'entraide et au service de remplacement (Rubin et al., 2005 ; Tipples et al., 2007). La composition de la main-d'œuvre influence également les solutions adoptées par les éleveurs. Olaizola et al. (2008) montrent que des équipements pour l'alimentation des troupeaux sont utilisés dans des exploitations avec du salariat. Mais les liens entre collectif de travail et solutions restent encore peu explicités.

Nous formulons l'hypothèse que la composition du groupe de travailleurs permanents qui assure et organise le travail de l'exploitation (appelé noyau organisateur) joue sur les voies d'adaptation choisies par les éleveurs laitiers. L'objectif de cette étude est ainsi d'identifier, dans des exploitations laitières du Ségala, les combinaisons possibles entre des solutions travail, les relations possibles avec la conduite de l'élevage, les types de collectifs de travail et les attentes des éleveurs. Dans une première partie nous présenterons le dispositif de l'étude mis en place avec les partenaires du développement dans cette région (Institut de l'élevage et organismes du contrôle laitier), puis nous aborderons les résultats concernant : (i) la diversité des collectifs de travail en élevage laitier et des solutions mises en place pour organiser le travail, (ii) les interactions entre collectifs de travail, structures et fonctionnement des exploitations. 


\section{Matériel et méthodes}

\section{Le Ségala, au sud du Massif Central}

Létude a été réalisée dans le Ségala, petite région agricole du sud du Massif Central en France qui concerne les départements de l'Aveyron, du Cantal et du Lot. Le milieu naturel est accidenté avec une altitude moyenne de $650 \mathrm{~m}$, variant de 300 à $850 \mathrm{~m}$. Les agriculteurs y représentent le quart de la population active, contre moins de 2,7 \% en France. Le tissu laitier est dense (100 à 120000 litres par km²) et composé de petites exploitations relativement intensives par rapport à d'autres régions de moyenne montagne française (Pflimlin et al., 2005). La main-d'œuvre repose essentiellement sur la famille. Les exploitations comportent en moyenne 36 vaches laitières et 60 ha de surface agricole utile (SAU) (Begon et al., 2009). La conduite de l'alimentation de l'atelier laitier est basée sur le maïs ensilage (19\% de la surface fourragère principale $[\mathrm{SFP}]$ ) ainsi que l'utilisation de prairies permanentes et temporaires pour la fauche et la pâture. Le chargement est élevé $(1,4$ unités gros bovins [UGB]/ha). La production laitière est de 6700 litres/vache/an en moyenne.

\section{Des enquêtes en élevage}

Le dispositif d'étude basé sur des enquêtes en élevage, a été construit avec les responsables des contrôles laitiers et l'Institut de l'élevage, partenaires professionnels de cette étude. Ces deux organismes étaient en effet largement concernés par la nécessité d'accompagner les éleveurs dans leur réflexion sur l'évolution de leur exploitation du point de vue du travail. Ils souhaitaient, par une meilleure connaissance des leviers mobilisés par les éleveurs faire face à des questions de travail et pouvoir améliorer leur appui aux éleveurs. Il leur semblait en effet nécessaire pour être plus pertinents dans leur offre de conseil, de connaître la diversité des options possibles mais aussi de prendre en compte la situation de chaque exploitation : attentes en termes de travail des éleveurs, nature du collectif de travail et le type de conduite de l'élevage.

L'entretien directif abordait : (i) le statut juridique, les équipements et les surfaces, (ii) la place du travail dans les préoccupations des éleveurs, (iii) la composition du collectif de travail, c'est-à-dire tous les travailleurs intervenant sur l'exploitation et (iv) les solutions « travail » mobilisées par les éleveurs, et les raisons de leurs choix. Pour décrire le collectif de travail, nous partons du principe que les travailleurs ne sont pas équivalents de par leur fonction dans le collectif, leur rythme de présence (Allaire, 1988) et le type de contrepartie à leur participation au travail (Madelrieux et Dedieu, 2008), et que les liens familiaux entre membres jouent sur le fonctionnement de celui-ci. Lors de l'enquête, tous les travailleurs intervenant sur l'exploitation ont ainsi été recensés et décrits par leur âge, sexe, statut juridique, rythme d'implication dans le travail sur l'exploitation (permanent, saisonnier...) et relations entre les personnes (familiales ou non). Deux catégories de main-d'œuvre sont identifiées : (i) le noyau organisateur comprend les travailleurs permanents pour 
qui l'activité agricole est prépondérante en temps et en revenu, et qui organisent le travail de l'exploitation (individuels, couples, associations), (ii) la main-d'œuvre hors noyau organisateur comprend toutes les autres personnes (bénévolat, entraide, salariat, prestataires de services).

Une liste de solutions sur le travail, relevant de trois ensembles (Dedieu et Servière, 2001) : la recomposition du collectif de travail, l'amélioration des bâtiments et des équipements et la conduite du troupeau et des surfaces, ainsi que les raisons de leur mise en œuvre a été construite en collaboration avec les partenaires professionnels, en utilisant des travaux réalisés dans d'autres régions françaises (Moreau et al., 2004). Le recueil des informations sur les solutions a utilisé cette base en laissant la possibilité de l'incrémenter quand nécessaire lors de l'enquête (solution ou raison non répertoriées initialement). Ces informations ont été complétées par des données, issues des relevés mensuels réalisés par les organismes du contrôle laitier (base de données Optilait), sur les dimensions de l'exploitation et le fonctionnement de l'élevage.

Les entretiens ont été réalisés lors de visites d'exploitations par les techniciens des contrôles laitiers de l'Aveyron, du Cantal et du Lot en 2006, et ont duré de 30 minutes à une heure. Ces enquêtes ont été conduites sur une période de trois mois (mai à juin 2006). Après cette période, 458 éleveurs ont été enquêtés parmi les 1600 adhérents au contrôle laitier, qui représentent $65 \%$ des éleveurs et $80 \%$ de la production laitière de la zone. La population enquêtée représente donc $19 \%$ des éleveurs laitiers de la zone. L'objectif était d'obtenir un échantillon le plus exhaustif possible de la population d'éleveurs adhérents au contrôle laitier.

Les élevages enquêtés se situent dans la moyenne des exploitations laitières du Ségala, avec un quota de 224000 litres/an, 36 vaches laitières et 57 ha de surface agricole utile (SAU) (Tab. 1). La SAU est majoritairement consacrée aux troupeaux. Les exploitations reposent sur un système fourrager mixte : le maïs ensilage occupe $17 \%$ de la surface fourragère principale (SFP), les prairies permanentes $33 \%$ et les prairies permanentes $50 \% .37 \%$ des exploitations sont spécialisées en élevage laitier. Les structures des exploitations sont relativement similaires entre les trois départements.

\section{Analyse des données}

La démarche d'analyse s'est déroulée en deux phases. La première a consisté en la construction de variables relatives aux exploitations (structure, conduite de l'élevage, localisation) (Tab. 1), les noyaux organisateurs (Tab. 2), les solutions sur le travail (Tab. 3), les raisons de mise en œuvre de ces dernières (Tab. 4) et en l'analyse descriptive de ces variables sur l'échantillon. Quatre types de noyaux organisateurs ont été définis dans l'échantillon (Tab. 2) selon le nombre de travailleurs et les relations entre eux (familiales ou non). Les " individuels " correspondent aux éleveurs seuls (182 exploitations). Les couples (61 exploitations) désignent les cas où les deux époux travaillent sur l'exploitation : la femme n'est pas toujours installée officiellement mais réalise au moins l'équivalent d'un travail à mi-temps. Les associations 
familiales (199 exploitations) sont gérées par plusieurs membres de la famille : groupement agricole d'exploitation en commun (GAEC) père-fils ou entre frères et sœurs. Les associations non familiales sont des regroupements d'exploitations ou d'exploitants hors cadre familiaux, où les associés n'appartiennent pas tous à la même famille (13 exploitations). À partir des enquêtes, 46 solutions de travail ont été répertoriées, et ont été codifiées en 20 variables (Tab. 3) qui regroupent des solutions relevant du même registre. Par exemple, la monotraite saisonnière et la monotraite annuelle ont constitué une variable "monotraite annuelle ou saisonnière ". Les solutions de travail ont été classées en trois groupes selon qu'elles concernaient la simplification de la conduite technique du troupeau et des surfaces, la recomposition de la main-d'œuvre ou l'amélioration des bâtiments et des équipements (Dedieu et Servière, 2001).

Tab 1. Caractéristiques des 458 élevages laitiers dans le Ségala.

\begin{tabular}{|c|c|c|c|c|}
\hline & & & $\begin{array}{c}458 \\
\text { exploitations } \\
\text { enquêtées }\end{array}$ & $\begin{array}{c}1600 \\
\text { exploitations au } \\
\text { Contrôle laitier }\end{array}$ \\
\hline \multirow{8}{*}{ Structure } & \multicolumn{2}{|c|}{ Élevages spécialisés en lait (\%) } & 37 & 45 \\
\hline & \multicolumn{2}{|l|}{ Vaches $(\mathrm{Nb})$} & 36 & 36 \\
\hline & \multicolumn{2}{|l|}{ SAU (ha) } & 57 & 60 \\
\hline & \multicolumn{2}{|l|}{ SFP (ha) } & 48 & 50 \\
\hline & \multicolumn{2}{|c|}{ Quota (1 000 L) } & 224 & 217 \\
\hline & \multirow{3}{*}{ Stabulation } & $\begin{array}{l}\text { Entravée } \\
(\%)\end{array}$ & 14 & l \\
\hline & & $\begin{array}{l}\text { Aire paillée } \\
(\%)\end{array}$ & 45 & l \\
\hline & & $\begin{array}{l}\text { Logettes } \\
(\%)\end{array}$ & 41 & l \\
\hline \multirow{4}{*}{$\begin{array}{l}\text { Conduite } \\
\text { de l'élevage }\end{array}$} & \multicolumn{2}{|c|}{ Maïs dans SFP (\%) } & 17 & 19 \\
\hline & \multicolumn{2}{|c|}{ Production laitière (L/vache/an) } & 6763 & 6727 \\
\hline & \multicolumn{2}{|c|}{ Chargement (UGB/an) } & 1,31 & 1,43 \\
\hline & \multicolumn{2}{|c|}{$\begin{array}{l}\text { Quantité de concentre } \\
\text { (kg/vache/an) }\end{array}$} & 1347 & l \\
\hline \multirow{3}{*}{ Département } & \multicolumn{2}{|l|}{ Aveyron (\%) } & 50 & 44 \\
\hline & \multicolumn{2}{|l|}{ Lot $(\%)$} & 23 & 25 \\
\hline & \multicolumn{2}{|l|}{ Cantal (\%) } & 27 & 11 \\
\hline
\end{tabular}


La deuxième étape a consisté à identifier des combinaisons particulières entre des solutions sur le travail et les relier aux systèmes décrits par la structure des exploitations, la conduite de l'élevage, le type de noyau organisateur et les raisons de mise en œuvre des solutions. Pour cela, une analyse des correspondances multiples (ACM) suivie d'une classification ascendante hiérarchique $(\mathrm{CAH})$ a été réalisée : chaque exploitation étant décrite par la présence/absence des vingt solutions (Tab. 3). Le logiciel SPAD a été utilisé pour réaliser les analyses statistiques. Les profils obtenus ont été décrits en utilisant les informations relatives aux structures et fonctionnement des élevages et aux noyaux organisateurs. Les différences entre profils ont été testées statistiquement (i) pour les variables quantitatives à l'aide d'analyses de variance et tests de Student-Newman-Keuls, et (ii) pour les variables qualitatives à l'aide de tests du $\chi^{2}$ et analyse des résidus ajustés.

Tab 2. Caractéristiques des noyaux organisateurs (NO) dans les 458 élevages laitiers.

\begin{tabular}{|c|c|c|c|c|c|c|}
\hline $\begin{array}{c}\text { Types } \\
\text { de noyau } \\
\text { organisateur } \\
\text { (NO) }\end{array}$ & $\begin{array}{c}\% \\
\text { élevages } \\
(\mathbf{n b} \\
\text { élevages) }\end{array}$ & $\begin{array}{c}\mathrm{Nb} \text { de } \\
\text { travailleurs } \\
\text { (écart-type) }\end{array}$ & $\begin{array}{l}\text { Âge du } \\
\text { plus jeune } \\
\text { travailleur } \\
\text { dans le } \\
\text { NO }\end{array}$ & $\begin{array}{c}\text { Présence d'un } \\
\text { travailleur } \\
\text { proche de } \\
\text { la retraite } \\
\text { (> } 55 \text { ans) } \\
\text { (\% des NO } \\
\text { concernés) }\end{array}$ & $\begin{array}{c}\text { Présence } \\
\text { d'activités } \\
\text { non } \\
\text { agricoles } \\
\text { (\% des NO } \\
\text { concernés) }\end{array}$ & $\begin{array}{l}\text { Recours } \\
\text { à des } \\
\text { bénévoles } \\
\text { (\% des NO } \\
\text { concernés) }\end{array}$ \\
\hline Individuel & 41 (188) & $1(0)$ & $42(7,5)$ & 7 & 0 & 51 \\
\hline Couple & $13(60)$ & $2(0)$ & $44(5,9)$ & 12 & 25 & 33 \\
\hline $\begin{array}{l}\text { Association } \\
\text { familiale }\end{array}$ & $44(202)$ & $2,4(0,7)$ & $37(8,2)$ & 31 & 14 & 30 \\
\hline $\begin{array}{c}\text { Association } \\
\text { non } \\
\text { familiale }\end{array}$ & $2(9)$ & $3,4(1,3)$ & $35(6,8)$ & 36 & 8 & 15 \\
\hline Total & 100 & $1,8(0,9)$ & $40(8,2)$ & 19 & 10 & 39 \\
\hline
\end{tabular}

Tab 3. Les 20 solutions « travail » dans les 458 élevages laitiers.

\begin{tabular}{llcc}
\hline Variable & \multicolumn{1}{c}{ Nom } & $\begin{array}{c}\text { \% élevages } \\
(\mathbf{n = 4 5 8 )}\end{array}$ & Leviers \\
\hline Entr & Entraide entre agriculteurs & 88 & MO \\
Prest & Recours à des prestataires de services avec salarié & 85 & MO \\
Augpat & Augmentation de la part d'herbe pâturée & 53 & COND \\
Equipnet & Équipement nettoyage & 48 & EQUIP \\
\hline
\end{tabular}




\begin{tabular}{|llll}
\hline Variable & \multicolumn{1}{c}{ Nom } & $\begin{array}{c}\text { \% élevages } \\
(\mathbf{n}=458)\end{array}$ & Leviers \\
\hline Cuma & $\begin{array}{l}\text { Matériel en Cuma (Coopérative d'utilisation } \\
\text { du mmatériel agricole })\end{array}$ & 46 & EQUIP \\
\hline EquipT & Équipement traite & 42 & EQUIP \\
\hline Bati & Aménagement et construction de bâtiments & 38 & EQUIP \\
\hline Chien & Chien de troupeau & 36 & COND \\
\hline SimpTS & Simplification du travail de saison & 35 & COND \\
\hline SR & Salariat & 31 & MO \\
\hline RC & Distribution d'une ration complète & 29 & COND \\
\hline DAC & Distributeur automatique de concentrés & 26 & EQUIP \\
\hline LS & Libre service & 18 & EQUIP \\
\hline Nbdistri & $\begin{array}{l}\text { Diminution du nombre de distribution } \\
\text { de la ration }\end{array}$ & 18 & COND \\
\hline Veau & Simplification de l'alimentation des veaux & 11 & COND \\
\hline MonoT & Monotraite annuelle ou saisonnière & 10 & COND \\
\hline 13T & Suppression d'une traite hebdomadaire & 8 & COND \\
\hline AchatF & Achat de fourrages à l'extérieur & 6 & COND \\
\hline FermSdT & Fermeture de la salle de traite & 5 & COND \\
\hline AchatG & Achat des génisses & 5 & COND \\
\hline
\end{tabular}

$M O$ : recomposer la main-d'cuvre ; EQUIP : améliorer les bâtiments et les équipements ; COND : simplifier la conduite du troupeau et des surfaces.

\section{Tab 4. Les raisons de mises en place des solutions travail.}

\begin{tabular}{|ccc|}
\hline Raisons de mise en ouvre des solutions travail & Nom variables & \% élevages \\
\hline Diminuer la durée du travail au quotidien & DimTA & 93 \\
\hline Gérer les pointes de travail & Pointe & 91 \\
\hline Diminuer la pénibilité physique du travail & Pénib & 74 \\
\hline Sécuriser le fonctionnement de l'exploitation & Sécur & 65 \\
\hline Assurer la pérennité de l'exploitation & Péren & 40 \\
\hline Se dégager des week-ends et des congés & Wecong & 32 \\
\hline Raisons économiques/Raisons techniques & Tech/éco & 28 \\
\hline Se libérer régulièrement une soirée & Soirée & 23 \\
\hline
\end{tabular}




\section{Résultats}

\section{La fin d'un modèle basé sur le couple d'exploitants}

Les résultats montrent que le modèle d'exploitation gérée par un couple, base du développement agricole depuis les années 1960, n’est plus la référence unique. Ces exploitations représentent moins de $15 \%$ de l'échantillon d'exploitations laitières. La famille reste cependant la base de $85 \%$ des noyaux organisateurs. Les sociétés entre associés non familiaux sont encore largement minoritaires (seulement $2 \%$ de l'échantillon). Dans $90 \%$ des exploitations, les membres du noyau organisateur travaillent sur l'exploitation toute l'année. Le vieillissement du noyau organisateur, lié aux préoccupations de transmission des exploitations, est plus marqué dans les exploitations individuelles ou les couples. En effet, ces deux types de noyau organisateur comprennent les travailleurs les plus âgés. La tendance est générale : $19 \%$ des élevages ont une personne du noyau organisateur de plus de 55 ans (Tab. 2). Cette question semble moins prégnante pour les associations qui comportent aussi la plus forte proportion de jeunes. Le bénévolat, en grande majorité familial (parents retraités, enfants), reste essentiel car $39 \%$ des noyaux organisateurs y ont recours (Tab. 2).

\section{Une diversité des solutions travail}

Le registre «main-d'œuvre » domine car $95 \%$ des exploitations de l'échantillon ont mis en place une ou plusieurs solutions jouant sur ce levier (Tab. 3). Les solutions les plus répandues sont le recours à de l'entraide entre agriculteurs pour réaliser des travaux agricoles $(88 \%)$ et à des prestataires de services, telles que la coopérative d'utilisation du matériel en commun (CUMA) avec chauffeur et l'entreprise de travaux agricoles (85\%). $31 \%$ des exploitations emploient un salarié sous différentes formes : salarié permanent, service de remplacement ou groupement d'employeurs. Un tiers d'entre elles recourt à de la main-d'œuvre salariée, notamment sous forme d'emploi collectif (groupement d'employeurs) ou prestation de service (CUMA, services de remplacement). Le salariat permanent concerne seulement $3 \%$ des élevages. Les exploitants seuls et les couples font davantage appel au salariat que les associations. L'amélioration des équipements et des bâtiments reste une voie importante d'adaptation des élevages puisque $83 \%$ des éleveurs y ont fait appel. Les éleveurs ont investi dans du matériel pour alimenter le troupeau comme le distributeur automatique de concentrés (26\%). L’amélioration du matériel a aussi porté sur le nettoyage des stabulations ( $48 \%$ ) (pailleuse, racleur automatique, etc.). $46 \%$ des exploitations ont recours à du matériel en CUMA pour les travaux sur les surfaces (SFP, cultures...). Les solutions de simplification de la conduite du troupeau et des surfaces sont diverses et concernent différents postes : l'alimentation, la traite, la reproduction et les surfaces fourragères. Elles concernent l'alimentation du troupeau laitier avec la distribution d'une ration complète $(29 \%)$, la réduction du nombre de distribution de la ration (18\%), l'allaitement des veaux laitiers (11\%) par exemple 
en alimentant les veaux une fois par jour. Les solutions touchant la traite concernent la monotraite annuelle ou saisonnière (10\%), la suppression d'une traite hebdomadaire en particulier la traite du dimanche soir $(8 \%)$ ou la fermeture de la salle de traite à une période de l'année $(5 \%)$. Seuls, quelques éleveurs achètent des génisses à d'autres éleveurs (5\%). Un nombre important d'éleveurs (53\%) dit avoir adapté la conduite du pâturage en augmentant la part d'herbe pâturée ou en diminuant la part de maïs. Les solutions ont également concerné les surfaces fourragères (35\%), par exemple avec le semis direct.

Les éleveurs déploient un nombre important de solutions (6,4 en moyenne). La réduction des volumes de travail au quotidien $(93 \%)$ et la gestion des pointes de travail $(91 \%)$ sont les raisons citées le plus fréquemment par les éleveurs pour expliquer l'adoption de solutions (Tab. 4). Diminuer la pénibilité physique du travail est également importante car elle est citée par $74 \%$ des éleveurs. Ils recherchent davantage de temps libre lors de soirées (23\%) ou de week-ends et congés (32\%). Les solutions ne répondent pas aux mêmes attentes (Fig. 1) : pour réduire leur temps de travail au quotidien, les éleveurs privilégient la simplification de la conduite technique ainsi que l'amélioration des équipements ou des bâtiments. La modification des rythmes de travail, que ce soit la réduction des pointes de travail, la libération d'une soirée ou la prise de congés, passe par des solutions d'adaptation portant sur la conduite technique ou la main-d'œuvre. La mécanisation diminue la pénibilité de certaines tâches, notamment le paillage des stabulations.

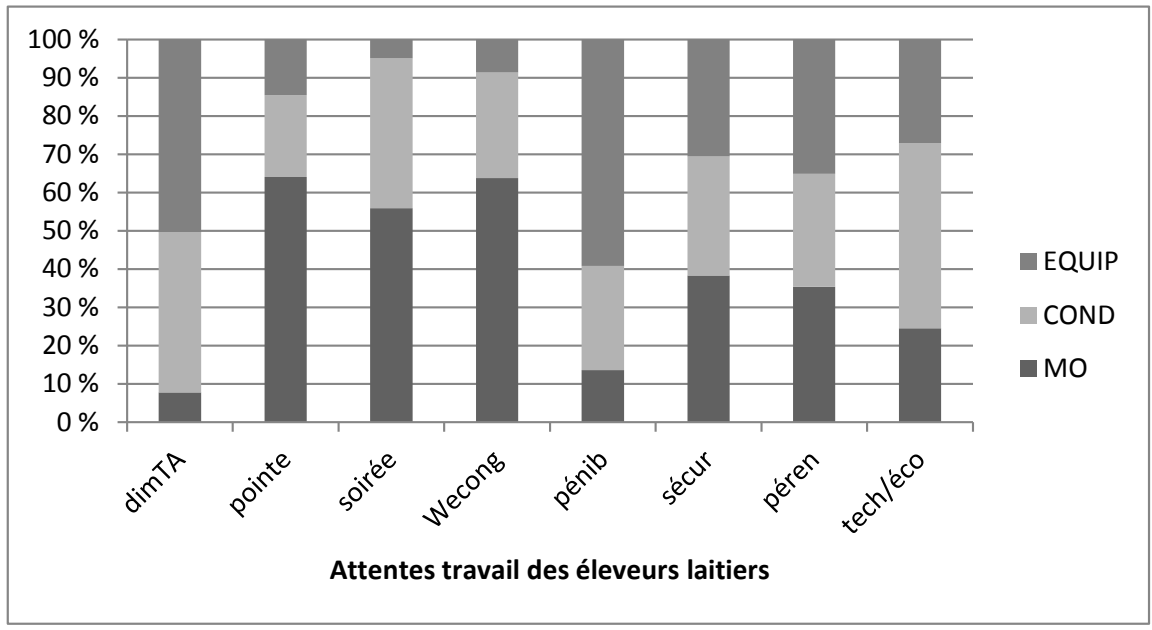

$M O$ : recomposer la main-d'cuvre; EQUIP : améliorer les bâtiments et les équipements. COND : simplifier la conduite du troupeau et des surfaces. Pour les noms des variables se reporter au tableau 4.

Fig.1. Relations entre attentes des éleveurs laitiers pour leur travail et groupes de solutions. 


\section{Six profils d'adaptation}

La classification des exploitations selon les cinq premiers axes issus de l'ACM a permis d'identifier six profils d'adaptation différents c'est-à-dire combinant de manière spécifique des solutions (Tab. 5) avec les structures et le fonctionnement de l'exploitation (Tab. 6 et 7).

Le profil 1 " pas de solution " $(\mathrm{n}=63)$ est caractérisé par le très faible recours à des solutions pour adapter le travail (3,6 en moyenne) (Tab. 5). Le système fourrager est basé sur une faible part de maïs $(13,6 \%$ de la SFP) et une part importante de pâturage pour alimenter le troupeau. Le niveau de productivité est relativement faible avec une moyenne de 6500 1/vache/an et un chargement de 1,2 UGB/ha. Les structures sont celles utilisées traditionnellement dans la région Ségala avec une étable entravée et un transfert à lait pour la traite (Tab. 7). Les exploitations sont préférentiellement localisées dans le Cantal et gérées par des exploitants plutôt plus âgés et plus souvent en couple que la moyenne de l'échantillon (Tab. 9). Les éleveurs n’ont pas exprimé d'attentes particulières pour leur travail (Tab. 8).

Le profil 2 « entraide et délégation » $(\mathrm{n}=143)$ est caractérisé par la mobilisation de solutions sur la main-d'œuvre. Les éleveurs ont recours à l'entraide et à la délégation des travaux à une entreprise pour les surfaces fourragères afin de gérer les pointes de travail. Les structures de production sont petites avec un quota de 174000 litres, 29 vaches et 46 ha de SAU. La part de maïs dans la surface fourragère principale $(14,5 \%)$ et la productivité des vaches $(6580 \mathrm{l} / \mathrm{vache} / \mathrm{an})$ sont plus faibles que la moyenne de l'échantillon. Les équipements sont anciens avec une stabulation entravée et un transfert à lait pour la traite. Les éleveurs de ce profil sont les plus âgés et le plus souvent seuls sur leur exploitation.

Le profil $3(\mathrm{n}=93)$ est caractérisé par de "nombreuses solutions pour améliorer les bâtiments et les équipements ». Les éleveurs ont investi dans des équipements de nettoyage pour la stabulation (pailleuse, racleur automatique, etc.) et la salle de traite. Les exploitations comptent parmi les plus grosses structures avec un quota de plus de 251000 litres, 40 vaches et 62 ha de SAU, et présentent la plus forte productivité avec une part de maïs importante dans la SFP (18,9\%). Ces solutions se retrouvent plus fréquemment dans des exploitations gérées par des associations (Tab. 9), les éleveurs étant parmi les plus jeunes de l'échantillon. Les raisons expliquant ces solutions sont la volonté de gérer les pointes de travail et de réduire la pénibilité physique du travail. Les exploitations sont plus fréquemment localisées en Aveyron.

Le profil 4 « traite et alimentation et distributeur automatique de concentrés (DAC)» $(\mathrm{n}=40)$ est caractérisé par des solutions pour simplifier la traite et l'alimentation des vaches, et améliorer les équipements (DAC). En fin de lactation, c'est-à-dire à la fin du printemps ou en été, les éleveurs ferment la salle de traite pendant quelques semaines et/ou pratiquent la monotraite saisonnière. Ils diminuent également le nombre de distribution de la ration des vaches laitières et utilisent du lait yogourt pour les veaux. L'alimentation des animaux est automatisée pour faciliter la distribution. Les raisons de mise en place des solutions expriment 
une volonté de la part des éleveurs de se libérer du temps (week-ends, congés, soirées). Les exploitations sont dans la moyenne de l'échantillon quant à la part de maïs dans la SFP (16,9\%) et à la productivité (6 740 1/vache/an). Elles sont plus fréquemment localisées en Aveyron.

Le profil 5 "Suppression d'une traite hebdomadaire, service de remplacement et équipement " $(\mathrm{n}=44)$ est caractérisé par l'utilisation d'un nombre de solutions important (9,1 en moyenne). Les éleveurs simplifient la conduite technique du troupeau en adoptant la suppression de la traite du dimanche soir et font appel aux services de remplacement pour certains travaux. Ils ont modifié les équipements avec un distributeur automatique pour l'allaitement des veaux, ont aménagé leur installation de traite et leur stabulation avec des équipements de nettoyage (racleur automatique, pailleuse). Les raisons exprimées par les éleveurs pour justifier ces choix sont de se libérer du temps (soirée, week-end, congés) et de réduire la pénibilité physique. Les structures sont relativement grandes avec 39 vaches, 59 ha de SAU et un quota de $244000 \mathrm{l}$. Les niveaux de productivité sont parmi les plus élevés de l'échantillon avec un chargement de 1,47 UGB/ha. La part de maïs dans la surface fourragère principale est importante $(21,1 \%)$.

Le profil 6 " plusieurs solutions pour chacune des trois composantes " $(\mathrm{n}=75)$ est composé d'exploitations combinant plusieurs solutions pour chacun des trois leviers (8,6 solutions en moyenne). Les éleveurs ont recours à de nombreuses solutions relatives à la conduite des surfaces et du troupeau. Ils utilisent la ration complète pour alimenter les vaches laitières. Des activités comme l'élevage des génisses ou la production de fourrages sont délocalisées hors de l'exploitation. Le travail de saison sur les surfaces fourragères est simplifié (semis direct, sur-semis pour les prairies). Les éleveurs recomposent la main-d'œuvre avec l'emploi de salariés, l'entraide entre agriculteurs et la délégation à des entreprises de travaux agricoles pour les travaux de saison (ensilages de maïs et d'herbe, moissons). Ils ont investi dans des équipements de nettoyage et de traite. Ces solutions sont mises en œuvre pour diminuer la pénibilité physique du travail et sécuriser le fonctionnement de l'exploitation. Ces exploitations sont de grande taille (256 0001 de quota et 39 vaches laitières), plus productives que la moyenne (7 $000 \mathrm{l} /$ vache/an) et une surface de maïs importante (19,8 \% de maïs dans la SFP). Comme pour le profil 3, les éleveurs sont parmi les plus jeunes de l'échantillon et plus fréquemment en association (Tab. 9). 
Tab 5. Pourcentage des solutions travail mises en œuvre dans les 458 fermes laitières selon les six profils.

\begin{tabular}{|c|c|c|c|c|c|c|c|c|c|}
\hline Leviers & Variable & $\begin{array}{c}P 1 \\
(n=63)\end{array}$ & $\begin{array}{c}P 2 \\
(n=143)\end{array}$ & $\begin{array}{c}\text { P3 } \\
(n=93)\end{array}$ & $\begin{array}{c}\text { P4 } \\
(n=40)\end{array}$ & $\begin{array}{c}\text { P5 } \\
(\mathrm{n}=44)\end{array}$ & $\begin{array}{c}\text { P6 } \\
(n=75)\end{array}$ & Tous & $\begin{array}{c}\text { Significativité } \\
\text { pour le test } \\
\text { du } \chi^{2}\end{array}$ \\
\hline $\mathrm{MO}$ & Entra & $35^{-}$ & $100^{+}$ & $98^{+}$ & 80 & 98 & $99^{+}$ & 88 & $* * *$ \\
\hline $\mathrm{MO}$ & Prest & $33^{-}$ & $97^{+}$ & 91 & 90 & 77 & $96^{+}$ & 85 & $* * *$ \\
\hline $\mathrm{MO}$ & SR & 22 & 30 & $5^{-}$ & 20 & $75^{+}$ & $49^{+}$ & 31 & $* * *$ \\
\hline EQUIP & Equipnet & 41 & $13^{-}$ & $74^{+}$ & 38 & $73^{+}$ & $80^{+}$ & 48 & $* * *$ \\
\hline EQUIP & Cuma & $17^{-}$ & 45 & 45 & 35 & 59 & $72+$ & 46 & $* * *$ \\
\hline EQUIP & EquipT & $29-$ & $10^{-}$ & $68^{+}$ & 43 & $73^{+}$ & $64^{+}$ & 42 & $* * *$ \\
\hline EQUIP & Bati & $13^{-}$ & $28^{-}$ & $58^{+}$ & 38 & 41 & $49^{+}$ & 38 & $* * *$ \\
\hline EQUIP & $\mathrm{DAC}$ & $8^{-}$ & $13^{-}$ & $40^{+}$ & $50^{+}$ & $73^{+}$ & $5^{-}$ & 26 & $* * *$ \\
\hline EQUIP & LS & 11 & $31^{+}$ & $8^{-}$ & 20 & $34^{+}$ & $1^{-}$ & 18 & $* * *$ \\
\hline COND & Augpat & $37^{-}$ & $65^{+}$ & $14^{-}$ & 63 & 57 & $83^{+}$ & 53 & $* * *$ \\
\hline COND & Chien & $24^{-}$ & 40 & $17^{-}$ & 25 & 43 & $64^{+}$ & 36 & $* * *$ \\
\hline COND & SimpTS & $16^{-}$ & 29 & 29 & 30 & $59^{+}$ & $56^{+}$ & 35 & $* * *$ \\
\hline COND & $\mathrm{RC}$ & 27 & $4^{-}$ & $40^{+}$ & $15^{-}$ & 34 & $71^{+}$ & 29 & $* * *$ \\
\hline COND & Nbdistri & $8^{-}$ & $5^{-}$ & 22 & $35^{+}$ & 9 & $41^{+}$ & 18 & $* * *$ \\
\hline COND & Veau & $2-$ & $3-$ & $3^{-}$ & $23^{+}$ & $41^{+}$ & $20^{+}$ & 11 & $* * *$ \\
\hline COND & MonoT & 6 & $6^{-}$ & 1 - & $75^{+}$ & 7 & $3-$ & 10 & $* * *$ \\
\hline COND & $13 \mathrm{~T}$ & 3 & $3^{-}$ & $1-$ & 10 & $55^{+}$ & 3 & 8 & $* * *$ \\
\hline COND & AchatF & 6 & 8 & 2 & 3 & 0 & $13^{+}$ & 6 & * \\
\hline COND & FermSdT & 5 & $0^{-}$ & 2 & $43^{+}$ & 5 & 0 & 5 & $* * *$ \\
\hline COND & AchatG & 8 & 6 & 0 & 0 & 0 & $12^{+}$ & 5 & $*$ \\
\hline
\end{tabular}

$M O$ : recomposer la main-d'auvre; EQUIP : améliorer les bâtiments et les équipements ; COND : simplifier la conduite du troupeau et des surfaces.

* pvalue $<0,05 ;{ }^{* * *}$ pvalue $<0,001$.

Les valeurs en gras indiquent les cellules pour lesquelles les résidus ajustés sont significativement différents de 0 (seuil de $p<0,05$ ). Le signe indique alors si la valeur est plus élevée (+) que celle donnée par le modèle $\chi^{2}$ d'indépendance ou plus faible (-). 
Tab 6. Moyenne des variables quantitatives selon les profils.

\begin{tabular}{|c|c|c|c|c|c|c|c|c|}
\hline & P1 & P2 & P3 & P4 & P5 & P6 & Tous & $\begin{array}{l}\text { Significativité } \\
\text { pour l'Anova }\end{array}$ \\
\hline $\mathrm{Nb}$ élevages & 63 & 143 & 93 & 40 & 44 & 75 & 458 & \\
\hline $\begin{array}{l}\mathrm{Nb} \text { vaches } \\
\text { laitières }\end{array}$ & $35,3 \mathrm{bc}$ & $28,7 \mathrm{a}$ & $40,0 \mathrm{c}$ & $31,5 \mathrm{ab}$ & $38,8 \mathrm{bc}$ & $39,4 \mathrm{c}$ & 35 & $* * *$ \\
\hline SAU (ha) & $61,1 \mathrm{~b}$ & $46,0 \mathrm{a}$ & $62,2 \mathrm{~b}$ & $54,0 \mathrm{ab}$ & $58,6 \mathrm{~b}$ & $60,4 \mathrm{~b}$ & 55,9 & $* * *$ \\
\hline SFP (ha) & $53,4 \mathrm{~b}$ & 40,6 a & $53,5 \mathrm{~b}$ & $45,7 \mathrm{ab}$ & $50,1 \mathrm{ab}$ & $52,4 \mathrm{~b}$ & 48,5 & $* * *$ \\
\hline $\begin{array}{l}\text { Maïs dans } \\
\text { la SFP (\%) }\end{array}$ & $13,6 \mathrm{a}$ & $14,5 \mathrm{a}$ & $18,9 \mathrm{~b}$ & $16,9 \mathrm{ab}$ & $21,1 \mathrm{~b}$ & $19,8 \mathrm{~b}$ & 17,1 & $* * *$ \\
\hline $\begin{array}{l}\text { Quota } \\
\text { (1 } 000 \text { l/an) }\end{array}$ & $209 \mathrm{ab}$ & $174 \mathrm{a}$ & $251 \mathrm{c}$ & $198 \mathrm{ab}$ & $244 \mathrm{bc}$ & $256 c$ & 217 & $* * *$ \\
\hline $\begin{array}{l}\text { Production } \\
\text { (1/vache/an) }\end{array}$ & $6502 a$ & $6580 \mathrm{a}$ & $\begin{array}{c}6997 \\
b\end{array}$ & $\begin{array}{c}6740 \\
\mathrm{ab}\end{array}$ & $\begin{array}{c}6813 \\
a b\end{array}$ & $\begin{array}{c}6995 \\
b\end{array}$ & 6763 & $* *$ \\
\hline $\begin{array}{l}\text { Âge du plus } \\
\text { jeune travailleur } \\
\text { dans le noyau } \\
\text { organisateur }\end{array}$ & $41,8 \mathrm{~b}$ & $42,3 \mathrm{~b}$ & $37,7 \mathrm{a}$ & $39,9 \mathrm{ab}$ & $40,0 \mathrm{ab}$ & $37,6 \mathrm{a}$ & 40,1 & $* * *$ \\
\hline $\begin{array}{l}\mathrm{Nb} \text { de solutions } \\
\text { travail solutions }\end{array}$ & $3,6 a$ & $5,4 b$ & $6,3 c$ & $7,3 \mathrm{~d}$ & $9,1 \mathrm{e}$ & $8,9 \mathrm{e}$ & 6,4 & $* * *$ \\
\hline $\begin{array}{l}\text { Chargement } \\
\text { (UGB/ha) }\end{array}$ & $1,20 \mathrm{a}$ & $1,26 \mathrm{ab}$ & $1,38 \mathrm{c}$ & $1,22 \mathrm{ab}$ & $1,47 \mathrm{c}$ & $1,37 \mathrm{bc}$ & 1,31 & $* * *$ \\
\hline $\begin{array}{l}\text { Quantité de } \\
\text { concentrés } \\
\text { (kg/vache/an) }\end{array}$ & 1376 & 1300 & 1415 & 1331 & 1272 & 1373 & 1347 & $\mathrm{~ns}$ \\
\hline
\end{tabular}

Pour une même ligne, les moyennes avec la même lettre ne sont pas significativement différentes ns : pas significatif; ${ }^{* *}$ pvalue $<0,01 ;{ }^{* * *}$ pvalue $<0,001$. 
Tab 7. Pourcentage des élevages appartenant à chaque modalité pour les variables qualitatives illustratives selon les profils.

\begin{tabular}{|c|c|c|c|c|c|c|c|c|c|}
\hline & & P1 & P2 & P3 & P4 & P5 & P6 & Tous & $\begin{array}{c}\text { Significativité } \\
\text { pour le test } \\
\text { du } \chi^{2}\end{array}$ \\
\hline \multirow{3}{*}{$\begin{array}{l}\text { Stabulation } \\
(\%)\end{array}$} & Entravée & $29^{+}$ & 27 & $2^{-}$ & $10^{+}$ & $2^{-}$ & $1^{-}$ & 14 & \multirow{3}{*}{$* * *$} \\
\hline & Aire paillée & 41 & 42 & 47 & 45 & 43 & 53 & 45 & \\
\hline & Logettes & 30 & 31 & $51^{+}$ & $45^{+}$ & $55^{+}$ & 45 & 41 & \\
\hline \multirow{3}{*}{$\begin{array}{c}\text { Département } \\
(\%)\end{array}$} & Aveyron & $33^{-}$ & 47 & $65^{+}$ & $70^{+}$ & 55 & 40 & 50 & \multirow{3}{*}{$* * *$} \\
\hline & Cantal & $57^{+}$ & 26 & 22 & 23 & $11^{-}$ & 21 & 27 & \\
\hline & Lot & $10^{-}$ & 27 & $14^{-}$ & $8^{-}$ & 34 & $39^{+}$ & 23 & \\
\hline
\end{tabular}

ns, not significant; ${ }^{* *}$ pvalue $<0,01 ;{ }^{* * *}$ pvalue $<0,001$.

Les valeurs en gras indiquent les cellules pour lesquelles les résidus ajustés sont significativement différents de 0 (seuil de $p<0,05)$. Le signe indique alors si la valeur est plus élevée (+) que celle donnée par le modèle $\chi^{2}$ d'indépendance ou plus faible (-).

Tab 8. Pourcentage des raisons de mise en œuvre des solutions pour chaque profil.

\begin{tabular}{|c|c|c|c|c|c|c|c|c|}
\hline & P1 & P2 & P3 & P4 & P5 & P6 & Tous & significativité \\
\hline $\begin{array}{l}\text { Diminuer la durée } \\
\text { du travail au quotidien }\end{array}$ & 87 & $86^{-}$ & $98^{+}$ & 98 & 100 & $99^{+}$ & 93 & $* * *$ \\
\hline Gérer les pointes de travail & $62^{-}$ & $96^{+}$ & $97^{+}$ & 88 & 96 & $97^{+}$ & 91 & $* * *$ \\
\hline $\begin{array}{l}\text { Diminuer la pénibilité } \\
\text { physique du travail }\end{array}$ & $49-$ & $66^{-}$ & $84+$ & 68 & $91^{+}$ & $90^{+}$ & 74 & $* * *$ \\
\hline $\begin{array}{l}\text { Sécuriser } \\
\text { le fonctionnement } \\
\text { de l'exploitation }\end{array}$ & $40^{-}$ & 69 & 62 & 55 & 75 & $83^{+}$ & 65 & $* * *$ \\
\hline $\begin{array}{l}\text { Assurer la pérennité } \\
\text { de l'exploitation }\end{array}$ & 30 & 42 & 41 & 28 & 43 & 49 & 40 & ns \\
\hline $\begin{array}{l}\text { Se dégager des week-ends } \\
\text { et des congés }\end{array}$ & $21-$ & 28 & $14^{-}$ & $60^{+}$ & $59^{+}$ & 39 & 32 & $* * *$ \\
\hline $\begin{array}{l}\text { Raisons économiques/ } \\
\text { Raisons techniques }\end{array}$ & $14^{-}$ & 27 & 24 & 33 & $23^{+}$ & 51 & 28 & $* * *$ \\
\hline $\begin{array}{l}\text { Se libérer régulièrement } \\
\text { une soirée }\end{array}$ & 14 & 17 & $10^{-}$ & $48^{+}$ & $64^{+}$ & 20 & 23 & $* * *$ \\
\hline
\end{tabular}

ns, pas significatif; ${ }^{* * *}$ pvalue $<0,001$.

Les valeurs en gras indiquent les cellules pour lesquelles les résidus ajustés sont significativement différents de 0 (seuil de $p<0,05$ ). Le signe indique alors si la valeur est plus élevée $(+)$ que celle donnée par le modèle $\chi^{2}$ d'indépendance ou plus faible (-). 
Tab 9. Pourcentage de noyaux organisateurs pour chaque profil.

\begin{tabular}{|c|c|c|c|c|}
\hline Profil & $\begin{array}{c}\text { Individuel } \\
(41 \%)\end{array}$ & $\begin{array}{l}\text { Couple } \\
(13 \%)\end{array}$ & $\begin{array}{l}\text { Association } \\
\text { familiale } \\
(44 \%)\end{array}$ & $\begin{array}{c}\text { Association } \\
\text { non familiale } \\
(2 \%)\end{array}$ \\
\hline P1 & 34,9 & $20,6^{+}$ & 41,3 & 3,2 \\
\hline P2 & $53,1^{+}$ & 12,6 & $33,6^{-}$ & 0,7 \\
\hline P3 & $32,3^{-}$ & $6,5^{-}$ & $55,9^{+}$ & $5,4^{+}$ \\
\hline P4 & 50,0 & 15,0 & 35,0 & 0,0 \\
\hline P5 & 40,9 & 15,9 & 38,6 & 4,5 \\
\hline P6 & $30,7^{-}$ & 10,7 & $57,3^{+}$ & 1,3 \\
\hline
\end{tabular}

Test $d u \chi^{2}:$ pvalue $=0,002$.

Les valeurs en gras indiquent les cellules pour lesquelles les résidus ajustés sont significativement différents de 0 (seuil de $p<0,05)$. Le signe indique alors si la valeur est plus élevée (+) que celle donnée par le modèle $\chi^{2}$ d'indépendance ou plus faible (-).

\section{Discussion}

\section{Évolution des collectifs de travail dans les exploitations laitières familiales}

Les résultats issus de l'échantillon à visée représentative montrent des tendances d'évolution des collectifs de travail dans les exploitations laitières du Ségala. Le modèle basé sur le couple d'exploitants n'est plus la seule référence : si les collectifs restent très majoritairement familiaux, les formes sociétaires se développent (Agreste, 2011). La question de la transmission et du devenir des exploitations individuelles ou en couple se pose car les exploitants y sont plus âgés, alors que les associations peuvent compter sur une plus forte proportion de jeunes. Dans les exploitations, le bénévolat familial est encore bien présent. Ce constat pointe ainsi la fragilité d'un grand nombre d'exploitations, dont l'organisation du travail repose fortement sur cette main-d'œuvre. La part du salariat et des prestataires de service confirme les tendances d'évolution des collectifs de travail des exploitations, déjà rapportées par plusieurs études (Gambino et al., 2012).

\section{Des profils d'adaptation qui articulent les trois leviers: simplification de la conduite, réorganisation de la main-d'œuvre et rationalisation des équipements}

Les solutions ne sont pas associées de façon aléatoire dans les élevages. Notre étude a permi d'identifier des combinaisons particulières de solutions en élevage laitier. Elle met en avant que des solutions de simplification de la conduite de l'élevage 
sont associées à celles concernant la main-d'œuvre et/ou les équipements comme illustré par le profil 5 «suppression d'une traite hebdomadaire, service de remplacement et équipement " et le profil 6 " plusieurs solutions pour chacune des trois composantes ». Contrairement aux études précédentes qui focalisaient sur une solution particulière - par exemple le robot de traite (Rotz et al., 2003) ou le système pâturant (Parsons et al., 2004), cette étude illustre des interactions entre les différentes composantes du système d'élevage : la conduite technique, les équipements et le recours à de la main-d'œuvre extérieure à l'exploitation. Ce résultat implique que pour raisonner une innovation ou un changement technique dans les élevages, les interactions entre la conduite d'élevage, la main-d'œuvre et les investissements doivent être prises en compte.

\section{Des profils d'adaptation non indépendants des noyaux organisateurs}

Les résultats confirment que les profils d'adaptation dépendent de la composition du noyau organisateur, de sa taille ainsi que dans certains cas (profils 1 et 2) de l'âge des travailleurs. Les profils 3 " nombreuses solutions touchant l'amélioration des bâtiments et les équipements " et 6 "plusieurs solutions pour chacune des trois composantes ", manifestant une dynamique importante basée sur des investissements et/ou des modifications significatives de la conduite technique dans une volonté d'être productif, se rencontrent plus fréquemment dans des exploitations gérées par des associations d'exploitants.

L'étude va dans le sens de travaux montrant que des changements de techniques dépendent d'un ensemble de facteurs liés au collectif de travail tels que l'âge, le nombre de travailleurs, la répartition du travail entre famille et salarié (Solano et al., 2006). Elle permet de considérer les travailleurs non pas indépendamment les uns des autres mais bien la nature du collectif de travail dans son ensemble (Madelrieux et Dedieu, 2008). Nos résultats rejoignent également d'autres études (Seegers et al., 2006 ; Dufour et al., 2007 ; Martel et al., 2008) qui montrent que les attentes des éleveurs pour améliorer leurs conditions de travail sont à prendre en compte pour expliquer les changements de pratiques. Au sein de noyaux organisateurs avec plusieurs associés, la question des week-ends ou des congés n'apparaît pas comme une réelle préoccupation, car son fonctionnement et sa taille permettent une rotation entre les exploitants (Seegers et al., 2006). Plutôt focalisées sur l'efficacité de leur travail, ces exploitations investissent dans la fonctionnalité de leurs bâtiments, et dans des équipements d'élevage pour travailler plus vite, comme le démontre le profil 5 "suppression d'une traite hebdomadaire, service de remplacement et équipement ». Jouant sur des registres différents pour modifier leur travail, les éleveurs seuls ou en couple recourent plus souvent à des simplifications touchant la traite et le pâturage en misant conjointement sur le recours à de la main-d'œuvre non familiale. Ils expriment souvent d'autres priorités que la productivité du travail (Rault, 2006) : se libérer du temps quotidiennement pour profiter de leur vie de famille, prendre des week-ends, des congés ou travailler en autonomie. Du fait des évolutions et de 
la diversification de la main-d'œuvre exploitante (Bewley et al., 2001 ; Haugen et Blekesaune, 2005), la prise en compte de la composition du collectif de travail est importante pour comprendre et accompagner les transformations du travail et des changements induits dans l'organisation des élevages. Le collectif de travail doit être analysé par les fonctions des travailleurs, leur implication en temps et les contreparties de leur travail, et non pas uniquement comme des unités de travailleurs.

Cette étude a permis de donner aux organismes en charge du conseil, une meilleure connaissance des solutions mises en œuvre par les éleveurs pour adapter leur travail, des combinaisons entre solutions ainsi que des liens avec le collectif de travail et le fonctionnement des élevages. La description de la diversité des solutions a montré qu'elles n'étaient pas toutes mobilisées selon la même fréquence. Des solutions sont très liées à la conjoncture par exemple économique. L'augmentation du prix du lait est un facteur qui joue sur la mise en ouvre de la réduction du nombre de traite, qui induit une baisse de la production laitière par vache. Par exemple, l'augmentation du prix du lait aurait conduit des éleveurs à arrêter la monotraite annuelle. En termes d'équipement, le robot de traite pour se libérer de l'astreinte de la traite, se développe dans les élevages laitiers.

\section{Conclusion}

La résolution des problèmes de travail dans les exploitations laitières familiales est cruciale pour assurer le devenir de la filière laitière. Nos résultats suggèrent que l'adaptation des élevages à des questions de travail renvoie à des combinaisons de solutions différentes selon la composition de la main-d'œuvre : travailler seul, en couple, à plusieurs de la famille, ou encore entre associés externes. La main-d'œuvre doit se raisonner en termes de composition et de relations entre les travailleurs, et pas uniquement en termes d'unités de travailleurs. Ainsi, la recherche de solutions doit être raisonnée différemment pour chaque cas, en prenant en compte les caractéristiques propres de chaque exploitation (collectif de travail, structure, fonctionnement, etc.), ainsi que les objectifs, les attentes et les conceptions du travail des exploitants. Réfléchir à des solutions pour répondre aux attentes des éleveurs, qui viseront à améliorer leurs conditions de travail et la durabilité sociale des exploitations, doit donc aussi bien envisager l'introduction d'une technique nouvelle que concevoir un nouveau système d'élevage à partir de combinaisons complexes de solutions.

\section{Bibliographie}

Agreste Primeur, 2011. Recensement agricole 2010, premières tendances, 266.

Allaire, G., 1988. Itinéraires et identités professionnelles des travailleurs de l'agriculture, Inra Actes et Communications, 3, 175-211.

Barthez, A., 1986. Du labeur paysan au métier d'agriculteur : l'élaboration statistique en agriculture, Cahiers d'Économie et sociologie rurales, 3, 45-72. 
Begon, M., Pailleux, J.-Y., Joly, N., Lémery, B., Dedieu, B., 2009. Les chemins pour durer en élevage bovin laitier : diversité des logiques d'action sur le long terme en Ségala (Massif Central), in $16^{e s}$ Rencontres Recherches Ruminants, Paris, France, 105-108.

Bewley, J., Palmer, R.W., Jackson-Smith, D.B., 2001. Modeling milk production and labor efficiency in modernized wisconsin dairy herds, Journal of Dairy Science, 84, 705-716.

Cochet, H., 2008. Vers une nouvelle relation entre la terre, le capital et le travail, Terres Agricoles, 134, 24-29.

Cournut, S., Dedieu, B., 2005. Simplification des conduites d'élevages bovins laitiers, Cahiers Agricultures, 14, 541-547.

Dedieu, B., Servière, G., 2001. Organisation du travail et fonctionnement des systèmes d'élevage, in $8^{e s}$ Rencontres Recherches Ruminants, Paris, France, $245-$ 250.

Dufour, A., Hostiou, N., Cournut, S., Dedieu, B., 2007. Le travail en élevage laitier : des conceptions, des noyaux organisateurs et des profils d'organisation variés, in $14^{e s}$ Rencontres Recherches Ruminants, Paris, France, 385-388.

Ferris, C.-P., Frost, J.-P., Binnie, R.C., Patterson, D.C., 2006. Dairy cows performance and labour inputs associated with two silage feeding systems, Grass and Forage Science, 61, 304-314.

Gambino, M., Laisney, C., Vert, J. (Eds), 2012. Le monde en tendances. Un portrait social prospectif des agriculteurs, Centre d'études et de prospective, SSP, ministère de l'Agriculture, de l'Alimentation, de la Pêche, de la Ruralité et de l'Aménagement du territoire, La Documentation française.

Hadley, .L., Harsh, S.B., Wolf, C.A., 2002. Managerial and financial implications of major dairy farm expansions in Michigan and Wisconsin, Journal of Dairy Science, 85, 2053-2064.

Haugen, M.S., Blekesaune, A., 2005. Farm and off-farm work and life satisfaction among Norwegian Farm women, Sociologia Ruralis, 45, 73-85.

Hervieu, B., Purseigle, F., 2013. Sociologie des mondes agricoles, Paris, Armand Colin, Collection U. Kung, L., Demarco, J.R.S., Siebenson, L.N., Joyner, E., Haenlein, G.F.W., Morris, R.M., 1997. An evaluation of two management systems for rearing calves fed milk replacer, Journal of Dairy Science, 80, 25292533.

Madelrieux, S., Dedieu, B., 2008. Qualification and assessment of work organization in livestock farms, Animal, 2, 453-446.

Martel, G., Dourmad, J.-Y., Dedieu, B., 2008. Do labour productivity and preferences about work load distribution affect reproduction management and performance in pig farms? Livestock Science, 118, 96-117.

Moreau, J.-C., Seegers, J., Kling-Eveillard, F., 2004. Répondre à la préoccupation travail des éleveurs : compte-rendu du programme national 2003 financé par l'Onilait. $1^{\text {re }}$ partie : $1^{\text {er }}$ recueil d'expériences sur les solutions possibles en exploitation, Rapport, Institut de l'élevage, Paris, 37p. 
Olaizola, A.M., Chertouh, T., Manrique, E., 2008. Adoption of a new feeding technology in Mediterranean sheep farming systems : Implications and economic evaluation, Small Ruminant Research, 79, 137-145.

Parsons, R.L., Luloff, A.E., Hanson, G.D., 2004. Can we identify key characteristics associated with grazing-management dairy systems from data survey? Journal of Dairy Science, 87, 2748-2760.

Pflimlin, A., Perrot, C., Parguel, P., 2005. Diversité des systèmes laitiers en France et en Europe. Les atouts des zones défavorisées pour les produits de qualité, Fourrages, 184, 493-512.

Rault, G., 2006. Approche sociologique en élevage et questions sur le conseil agricole, Fourrages, 185, 29-35.

Rémond, B., Pomiès, D., 2007. Once-daily milking of Holstein cows for one-week decreases milk yield by twenty-five percent without any carry-over effect, Livestock Science, 110, 192-195.

Rotz, C.A., Coiner, C.U., Soder, K.J., 2003. Automatic milking systems, Farm size, and milk production, Journal of Dairy Science, 86, 4167-4177.

Rubin, B., Huchon, J.-C., Sabatté, N., Desarmenien, D., Gaboriau, L., Goulard, L., 2005. Robot de traite, monotraite, embauche d'un salarié : impacts sur le fonctionnement et les résultats d'exploitations laitières en Pays-de-la-Loire, Fourrages, 185, 63-72.

Seegers, J., Moreau, J.-C., Béguin, E., Guillaumin, A., Frappat, B., 2006. Attentes des éleveurs laitiers vis-à-vis de leurs conditions de travail et évolution de leurs systèmes d'exploitation, Fourrages, 185.

Solano, C., Leon, H., Pérez, E., Tole, L., Fawcett, R.H., Herrero, M., 2006. Using farmer decision-making profiles and managerial capacity as predictors of farm management and performance in Costa Rica dairy farms, Agricultural Systems, 88, 395-428.

Tipples, R., Verwoerd, N., Bewsell, D., Dalley, D., Turner, D., 2007. Social impacts of Once-a-Day milking, in Proceedings of the Once-a-Day Milking Conference, Tempero Centre, LIC, Newstead, Hamilton, pp. 23-27.

Wagner, A., Palmer, R.W., Bewley, J., Jackson-Smith, D.B., 2001. Producer Satisfaction, Efficiency, and Investment Cost Factors of Different Milking Systems, Journal of Dairy Science, 84, 1890-1898. 